\title{
Effect of Molecular Size on Corrosion Inhibition Efficiency of Some Alkyl Amines*
}

\author{
Kunitsugu ARAMAKI**
}

Many investigations concerning the correlation between inhibition effectiveness and molecular size of organic corrosion inhibitors have been reported. In most cases the inhibition efficiency increases with the molecular size for homologous series of the inhibitors. The efficiency of dialkylamines, for example, is closely related to area of metal surface which an amine molecule would effectively cover ${ }^{1)}$. This relationship has been represented for aliphatic amines by three separate curves for primary, secondary, and tertiary amines ${ }^{23}$. A linear correlation between the efficiency and cross-sectional area of some alkyl amines has been shown based on the assumption of an equal adsorption ability for each amines ${ }^{3)}$. Ayres and Hackerman ${ }^{4}$ have concluded, however, that this area is not of first order importance to the efficiency for pyridines. The inhibition efficiency of piperidine derivatives for cathodic partial reaction is hardly related to the molecular coverage area which is an effectively covered area per an inhibitor molecule adsorbed on metal, while the area is important to the efficiency for anodic partial reaction ${ }^{52,6)}$. Assuming the efficiency to be proportional to the area, a correlation of the anodic inhibition efficiency to the electron density at nitrogen atom and to the steric requirement for 2- and 6-substituted piperidines is successfully confirmed by application of an equation derived from the Ingold-Taft equation ${ }^{6}$. In this communication, the relationship between the efficiency and the molecular coverage area is examined for $n$-monoalkyl amines containing 2 to 5 carbon members. The adsorption abilities of these amines on both cathodic and anodic metal surface should be equal because of little differences in base strengths $\left(C_{2} 10.63 ; C_{3} 10.57 ; C_{4} 10.63\right.$; $\mathrm{C}_{5}$ 10.64) and the polar substituent constants $\left(\mathrm{C}_{2}\right.$ $0.880 ; \mathrm{C}_{3} 0.865 ; \mathrm{C}_{4} 0.850 ; \mathrm{C}_{5} 0.840$ ). Therefore, the extent of adsorption is presumed to be independent of the carbon number of these amines at given concent-

\footnotetext{
- Presented in part at the 38 th annual meeting of the Electrochemical Society of Japan (1971, Osaka)

** Department of Engineering, Keio University at Hiyoshi (665 Hiyoshi, Kohoku-ku, Yokohama)
}

rations.

Corrosion rates were determined by polarization of $99.89 \%$ iron electrode in $6.1 \mathrm{M} \mathrm{HCl}$ solution at $30^{\circ} \mathrm{C}$. Corrosion current $i_{\text {corr }}$ and cathodic and anodic partial current $i_{\mathbf{c}}$ and $i_{\mathbf{a}}$ were obtained by extrapolation of Tafel lines ${ }^{5}$. Total, cathodic, and anodic inhibition efficiency $I_{\mathrm{t}}, I_{\mathrm{c}}$, and $I_{\mathrm{a}}$ were calculated by $I_{\mathrm{t}}=1-i_{\text {corr }}$ $/ i^{\circ}{ }_{\text {corr }}, I_{\mathrm{c}}=1-i_{\mathrm{c}} / i^{\circ}{ }_{\text {corr }}$, and $I_{\mathrm{a}}=1-i_{\mathrm{a}} / i^{\circ}{ }_{\text {corr }}$, where $i^{0}{ }_{\text {corr }}$ refers to the corrosion current for uninhibited electrode. The molecular coverage areas were evaluated by using Stuart molecular models of protonated ammonium cation and unprotonated amine in the same manner as has been given ${ }^{\prime}$. Because of the possibility of free orientation of cation adsorbed on metal, the maximum area $A^{+} \max$ (parallel alkyl group to the surface), the minimum area $A^{+}{ }_{\min }$ (perpendicular), and the intermediate areas $A^{+}{ }_{30}$ and $A^{+}{ }_{60}$ of the cations of which alkyl trail is tilted to make angles of inclination at $30^{\circ}$ and $60^{\circ}$ with the surface were evaluated, respectively.

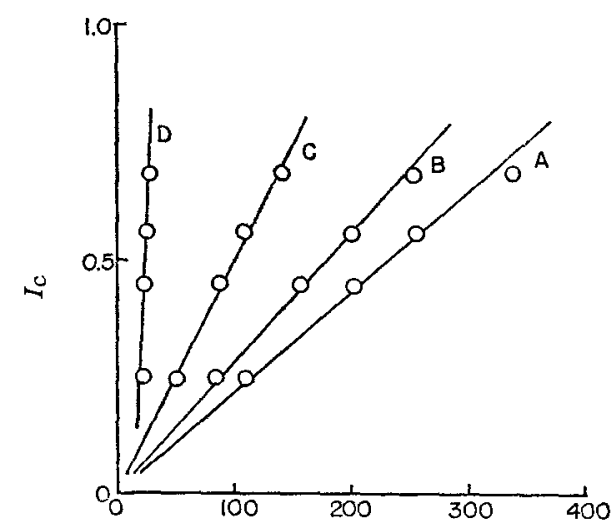

Molecular coverage area $\left(A^{2}\right)$

Fig. 1 Relationship between cathodic inhibition efficiency $I_{c}$ and molecular coverage areas of ammonium cation

$\mathrm{A}: A^{+}{ }_{\text {mex }}, \mathrm{B}: A^{+}{ }_{80}, \mathrm{C}: A^{+}{ }_{60}, \mathrm{D}: A^{+}{ }_{\text {min }}$ 
Fig. 1 shows linear relationships between the $I_{c}$ value at $0.01 M$ of the inhibitor concentration and the areas of the cation. The result disagrees with the previous result for piperidines that effect of the area on $I_{\mathrm{c}}$ is neglisible.

This can be caused by far larger differences in the areas of alkyl amines than piperidines and by more flexible alkyl trail than rigid piperidine ring. Assuming that these amines are alike in the extent of adsorption, and that the metal surface is physically covered by the organics for inhibiting the corrosion, the area should rather be reasonably related to the inhibition efficiency. This correlation was also con-

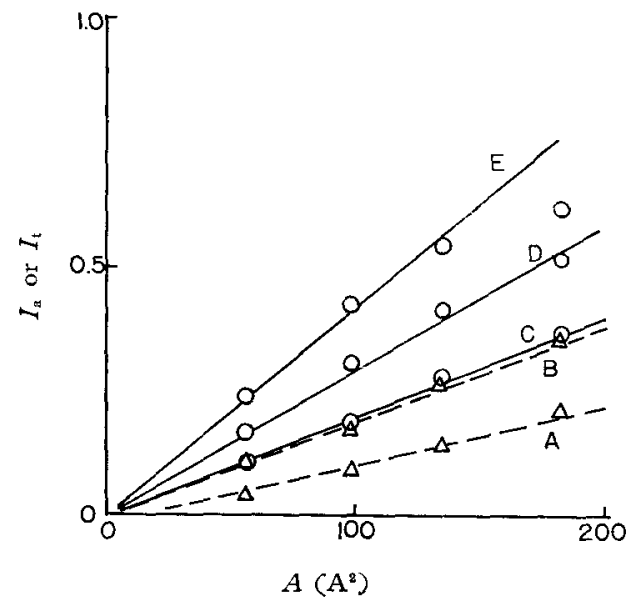

Fig. 2 Relationship of anodic and total inhibition efficiency $I_{\mathrm{a}}$ and $I_{\mathrm{t}}$ to molecular coverage area of amine $A$

A : $I_{a}$ at $0.01 M$ of the inhibitor concentration

$\mathrm{B}: I_{\mathrm{a}}$ at $0.03 \mathrm{M}, \mathrm{C}: I_{\mathrm{t}}$ at $0.003 \mathrm{M}$

$\mathrm{D}: I_{\mathrm{t}}$ at $0.01 \mathrm{M}, \mathrm{E}: I_{\mathrm{t}}$ at $0.03 \mathrm{M}$

firmed at the inhibitor concentrations less than 0.01 $M$.

The $I_{\mathrm{a}}$ values at 0.01 and $0.03 \mathrm{M}$ of the concentration were plotted against the molecular coverage area of free amine $A$ in Fig. 2, where points fall on straight lines. Being assumed to relate linearly with $A, I_{\mathrm{a}}$ for piperidines is given by

$$
\log \left(A_{\mathrm{o}} I_{\mathrm{a}} / A I_{\mathrm{ao}}\right)=\rho^{*} \Sigma \sigma^{*}+E_{\mathrm{s}}
$$

where zero subscripts refer to a reference compound and $\rho^{*}, \Sigma \sigma^{*}$, and $E_{\mathrm{s}}$ are the reaction, polar substituent, and steric substituent constants ${ }^{6}$. For alkyl amines, good agreement of this relationship was verified and the steric requirement for adsorption process was found to be of little importance to $I_{\mathrm{a}}$. It follows from the correlations of both $I_{c}$ and $I_{\mathrm{a}}$ to the areas that $I_{\mathrm{t}}$ relates proportionally to the area, as shown in Fig. 2. Al- though these amines are rather cathodic inhibitors, $I_{\mathbf{t}}$ is corralated well to the area of unprotonated amine adsorbed on metal by the formation of metal-nitrogen coordinate bond.

The linear correlation between the efficiency and the molecular coverage area is thus concluded to exist for alkyl amines which are alike in adsorption capacities and steric interactions. However, $I_{\mathrm{t}}$ higher than 0.6 deviates from the correlation. This is presumed to arise from decrease of the area due to change in the orientation of inhibitor molecule inclined to perpendicular toward the surface under the influence of lateral interaction between alkyl groups of adjacent molecules adsorbed on metal. Therefore, the linear relationship can be valid at low enough inhibition efficiency to cause the inhibitor to be adsorbed on the metal surface independently. These lines in the figures are supposed to curve at the high efficiency and to level off near 1.0 of the efficiency.

(Received Feb. 27, 1973)

\section{References :}

1) K. Aramaki. N. Hackerman, J. Electrochem. Soc. 115, 1007 (1968).

2) A.I. Altsybeeva, S.Z. Levin, A.P. Dorokhov, "Proceedings 3 rd European Symposium on Corrosion Inhibitors", p. 501 (1971), Universita Degli Studi di Ferrara.

3) S. Nakagawa, G. Hashizume, This Journal 36, 570 (1968).

4) R.C. Ayers, Jr., N. Hackerman, J. Electrochem. Soc. 110, 507 (1963).

5) K. Aramaki, This Journal 40, 566 (1972).

6) K. Aramaki, ibid 41, 550 (1973). 